CERN - PS DIVISION

CERN-PS 2001-002 (AE)

CLIC Note 469

\title{
Machine-Detector Interface at CLIC
}

\author{
D. Schulte
}

\begin{abstract}
The aim of the Compact Linear Collider (CLIC) study [1] is to design a highenergy linear $\mathrm{e}^{+} \mathrm{e}^{-}$collider. The effort is focused on an accelerator with a centreof-mass energy of $E_{c m}=3 \mathrm{TeV}$ and a luminosity of $\mathcal{L}=10^{35} \mathrm{~cm}^{-2} \mathrm{~s}^{-1}$. Careful study of the expected background in the interaction point (IP) of this machine is necessary. In CLIC, the background sources are expected to be more severe than in other studies like JLC, NLC and TESLA because of the higher luminosity, higher energy and stronger beam-beam effects. Coherent pair creation, which is almost completely absent at lower energies, will be very important. This paper gives an overview of the current status of the background calculations including the corresponding detector simulations.
\end{abstract}

Linear Collider Workshop, $24-28^{\text {th }}$ October

Fermi National Accelerator Laboratory

Batavia, U.S.A.

Geneva, Switzerland

19 January 2001 


\title{
Machine-Detector Interface at CLIC
}

\author{
D. Schulte \\ CERN, Geneva, Switzerland
}

\begin{abstract}
The aim of the Compact Linear Collider (CLIC) study [1] is to design a high-energy linear $\mathrm{e}^{+} \mathrm{e}^{-}$collider. The effort is focused on an accelerator with a centreof-mass energy of $E_{c m}=3 \mathrm{TeV}$ and a luminosity of $\mathcal{L}=10^{35} \mathrm{~cm}^{-2} \mathrm{~s}^{-1}$. Careful study of the expected background in the interaction point (IP) of this machine is necessary. In CLIC, the background sources are expected to be more severe than in other studies like JLC, NLC and TESLA because of the higher luminosity, higher energy and stronger beam-beam effects. Coherent pair creation, which is almost completely absent at lower energies, will be very important. This paper gives an overview of the current status of the background calculations including the corresponding detector simulations.
\end{abstract}

\section{INTRODUCTION}

The focus of this paper is on the luminosity spectrum in CLIC and on the background induced by beam-beam interaction. A preliminary design of the detector masking system is presented, together with first results of tracking the background through the detector. References [2-4] can serve as an introduction to this field. The minimum crossing angle and the beam delivery system are described because of their strong impact on the conditions for the experiment. The investigation of the background produced in the machine before or after the IP is not covered because it is not yet complete.

\section{BEAM DELIVERY SYSTEM}

The two main parts of a beam delivery system are the collimation system and the final focus system. The beam tails are scraped off in the former and the beam is focused to the required, extremely small, spot size in the latter. Currently, only a $3.2 \mathrm{~km}$ long design of the final focus system exists, which achieves $80 \%$ of the target luminosity [5]. The collimation system remains to be designed, including whether it can be partially integrated into the final focus system. The crossing angle necessary between the two beam lines is discussed later since it depends on the beam-beam effects. 
TABLE 1. Main parameters of CLIC at $E_{c m}=3 \mathrm{TeV}$. All backgrounds are per bunch crossing.

\begin{tabular}{|c|c|c|c|}
\hline Luminosity & $\mathcal{L}$ & {$\left[10^{34} \mathrm{~cm}^{-2} \mathrm{~s}^{-1}\right]$} & 10.0 \\
\hline repetition frequency & $f_{r}$ & {$[\mathrm{~Hz}]$} & 100 \\
bunches per train & $N_{b}$ & {$[\mathrm{~ns}]$} & 154 \\
distance between bunches & $\Delta_{b}$ & {$\left[10^{10}\right]$} & 0.67 \\
\hline particles per bunch & $N$ & {$[\mu \mathrm{m}]$} & 0.4 \\
bunch length & $\sigma_{z}$ & {$[\mu \mathrm{m}] /[\mu \mathrm{m}]$} & $0.68 / 0.02$ \\
emittances & $\gamma \epsilon_{x} / \gamma \epsilon_{y}$ & {$[\mathrm{~nm}] /[\mathrm{nm}]$} & $43 / 1$ \\
beam sizes & $\sigma_{x}^{*} / \sigma_{y}^{*}$ & {$[\%]$} & 31 \\
average energy loss & $\delta$ & & 2.3 \\
beamstrahlung photons per particle & $n_{\gamma}$ & {$\left[10^{3}\right] /\left[10^{6} \mathrm{GeV}\right]$} & $455 / 39$ \\
number/energy of incoh. pairs & $N_{\text {pairs }} / E_{\text {pairs }}$ & {$\left[10^{3}\right] /\left[10^{9} \mathrm{GeV}\right]$} & $1.4 \times 10^{6} / 440$ \\
number/energy of coh. pairs & $N_{\text {coh }} / E_{\text {coh }}$ & $N_{H}$ & 4.05 \\
hadronic events & $N_{H}$ &
\end{tabular}

Transverse jitters of the two beams at the IP are one of the most significant sources of luminosity loss. The possible performance of an intra-pulse interactionpoint feedback has been studied [6] for $E_{c m}=1 \mathrm{TeV}$; in principle the same performance is expected for $E_{c m}=3 \mathrm{TeV}$. It showed a reduction of the luminosity loss by a factor 3 . Recently, simulations of the luminosity with $\sqrt{s}>0.99 E_{c m}$ have been done for large relative offsets of the two beams. For $\Delta y=10 \mathrm{~nm} \approx 6 \sigma_{y}^{*}$, the feedback improves the achieved luminosity from $2.5 \%$ to $55 \%$, which corresponds to an uncorrected offset of $\Delta y=2 \sigma_{y}^{*}$.

\section{BEAM-BEAM EFFECTS}

The most important CLIC parameters are listed in Table 1 for different energies. In future linear colliders, the beams must be focused to very small spot sizes, to achieve the required high luminosity. As a consequence, the beam-beam forces will be very strong, which gives rise to high background levels. At $E_{c m}=3 \mathrm{TeV}$, each beam particle will radiate on average about $\delta=30 \%$ of its initial energy in the form of so-called beamstrahlung, a process similar to the emission of synchrotron radiation.

A large number of electron-positron pairs will be produced by the three incoherent processes $\gamma \gamma \rightarrow \mathrm{e}^{+} \mathrm{e}^{-}$, e $\gamma \rightarrow \mathrm{e}\left(\mathrm{e}^{+} \mathrm{e}^{-}\right)$and ee $\rightarrow \mathrm{ee}\left(\mathrm{e}^{+} \mathrm{e}^{-}\right)$, where the initial photons were produced by beamstrahlung. At high energies also coherent pair production will play an important role in CLIC. In this process, which is almost completely absent at lower energies, a beamstrahlung photon turns into an electronpositron pair in the strong coherent field of the oncoming bunch. At $E_{c m}=3 \mathrm{TeV}$, the number of produced particles is only six times smaller than the number of beam particles. All these effects are simulated using GUINEA-PIG [7].

Two colliding photons can also produce hadronic final states. In CLIC at $E_{c m}=$ $3 \mathrm{TeV}$ one expects 4.05 of these events per bunch crossing with a $\gamma \gamma$-centre-of-mass 

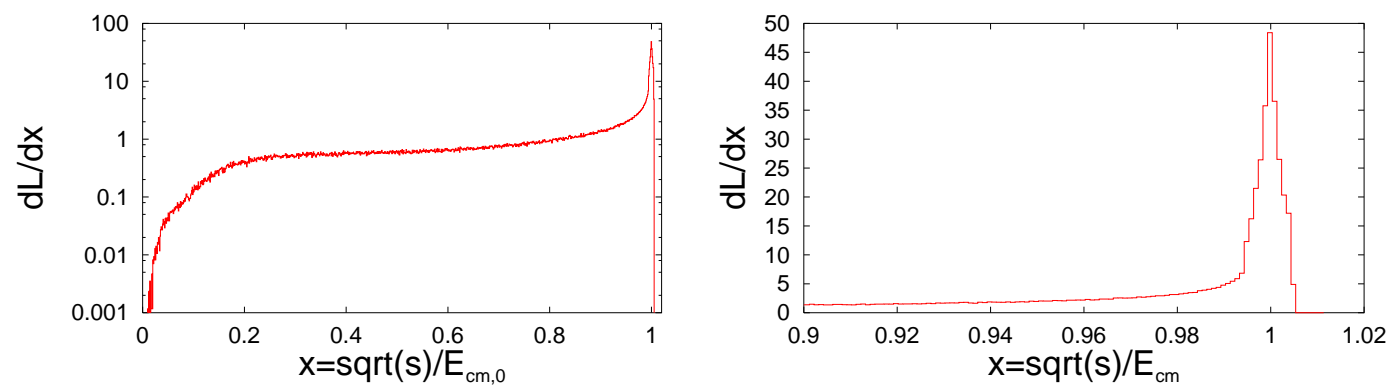

FIGURE 1. The luminosity spectrum in CLIC. Left the full spectrum, right the part close to the nominal centre-of-mass energy.

energy of $\sqrt{s_{\gamma \gamma}} \geq 5 \mathrm{GeV}$ [4]. The initial photons for these events have been created using GUINEA-PIG and the events have been simulated using PYTHIA. About 8000 of them are stored in a data base which can be accessed with the library HADES [8]. This allows to easily add hadronic background to the physics events, in order to estimate the effect.

\section{CROSSING ANGLE}

The total crossing angle between the two beam lines is currently fixed to $\theta_{c}=$ $20 \mathrm{mrad}$. So-called crab-crossing cavities are used to avoid the luminosity reduction that would otherwise result from the crossing angle.

Two effects set a lower limit to $\theta_{c}$. First, one has to avoid the multi-bunch kink instability. On its way from the final quadrupole to the IP, a bunch will have close encounters with the bunches of the other beam that have already collided. It can receive transverse kicks which in turn lead to an offset in the IP. At $E_{c m}=3 \mathrm{TeV}$, about half of the kick is due to the beam particles, the other half is due to the coherent pairs. To avoid initial offsets of the bunch train being amplified by this effect, a minimum crossing angle of about $\theta_{c}=20 \mathrm{mrad}$ is necessary.

Second, one has to minimise losses of the spent beam in the final quadrupole or masking system, because secondaries could be a hazard for the detector. The most important source of loss is the coherent pairs. To provide the necessary exit hole for the spent beam, a crossing angle of about $\theta_{c} \approx 20 \mathrm{mrad}$ is necessary [4].

\section{LUMINOSITY SPECTRUM}

The main effects that influence the luminosity spectrum of a linear collider are initial-state radiation, initial single-bunch energy spread, bunch-to-bunch or pulseto-pulse energy variations and beam-beam effects. Initial state radiation is an unavoidable QED effect that can in principle be calculated to any desired accuracy. The single-bunch energy spread mainly depends on the single-bunch beam loading in the main linac which is partially compensated by the accelerating RF. During 
acceleration, the energy spread is of the order of a few percent to ensure transverse beam stability. At the end of the linac it is reduced with the RF to a full width of $1 \%$ in order to fit into the energy acceptance of the final focus system. It could be reduced slightly more at the expense of a less stable beam at the end of the linac. The bunch-to-bunch energy variations are an order of magnitude smaller than the single-bunch energy spread [9]. Also pulse-to-pulse variations have to be kept to this low level, so these two effects can be neglected compared to the single bunch energy spread. In the case of CLIC at $E_{c m}=3 \mathrm{TeV}$, the main beambeam effects are beamstrahlung and coherent pair creation. Beamstrahlung will reduce the luminosity with $\sqrt{s}>0.99 E_{c m}$ to about $30 \%$ of the overall luminosity. The coherent pairs will add to the tail of the spectrum at low energies. Since the produced particles can fly either way, coherent pair production will also give rise to small $\mathrm{e}^{-} \mathrm{e}^{-}$and $\mathrm{e}^{+} \mathrm{e}^{+}$luminosities.

The result of simulations that include all effects except initial state radiation is shown in Fig. 1. It has been calculated with a modified version of GUINEAPIG. A library, CALYPSO [10], has been written that allows the inclusion of this luminosity spectrum in event generators. It also contains the luminosity spectra for all possible other collisions such as $\gamma \gamma, \mathrm{e}^{-} \gamma, \mathrm{e}^{+} \mathrm{e}^{+}$etc.

\section{DETECTOR SIMULATION}

Based on GEANT, a very simple detector simulation has been written to evaluate the background. It contains vertex detector, beam pipe, masking system, central tracker and calorimeter.

To avoid problems with the track reconstruction in the vertex detector, the density of charged hits has to stay below a few $\mathrm{mm}^{-2}$ per bunch train [11]. However, a limit of $1 \mathrm{~mm}^{-2}$ is adopted to have some margin. The incoherent pairs can produce a significant number of hits. Simulation of 100 bunch crossings show that for a main detector field of $B_{z}=4 \mathrm{~T}$, the acceptable hit level is reached for a radius $r_{v t x}=30 \mathrm{~mm}$ of the innermost layer of the vertex detector with an angle coverage of $|\cos \theta| \leq 0.98$. The backscattering of particles in the masks has been neglected in this simulation.

A preliminary design of the mask has been found. The outer mask, which protects the main tracker from secondary photons, consists of a conical part followed by a cylinder; both are made of tungsten, see Fig. 2. The distance between the IP and the opening of the mask is $z_{1}=1 \mathrm{~m}$. The inner opening angle $\theta_{i}$ has to be large enough to ensure that the bulk of the particles enters the mask. The envelope of

these particles can be approximated as $r=55 \mathrm{~mm} \sqrt{z / \mathrm{m}}$, see Fig. 3. This results in $\theta_{i}>55 \mathrm{mrad}$; in order to have some margin $\theta_{i}=80$ mradian is adopted. The outer angle, down to which the main detector extends, is $\theta_{m}=120 \mathrm{mrad}$.

The inner mask has to protect the vertex detector from backscattered low energy electrons and positrons [3]. These would be led directly into the vertex detector by the main detector field. It also shields the inner part of the detector from neutrons 


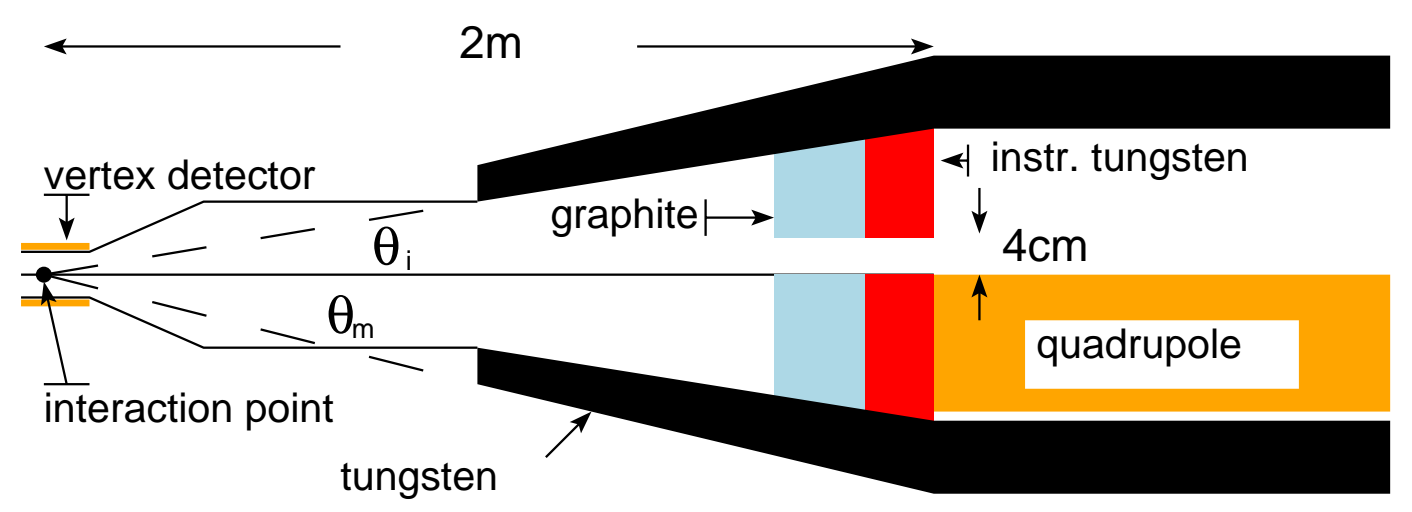

FIGURE 2. View from above on the adopted mask design. The sketch is stretched in vertical direction. Care has to be taken that no particles are backscattered through the hole in the mask.
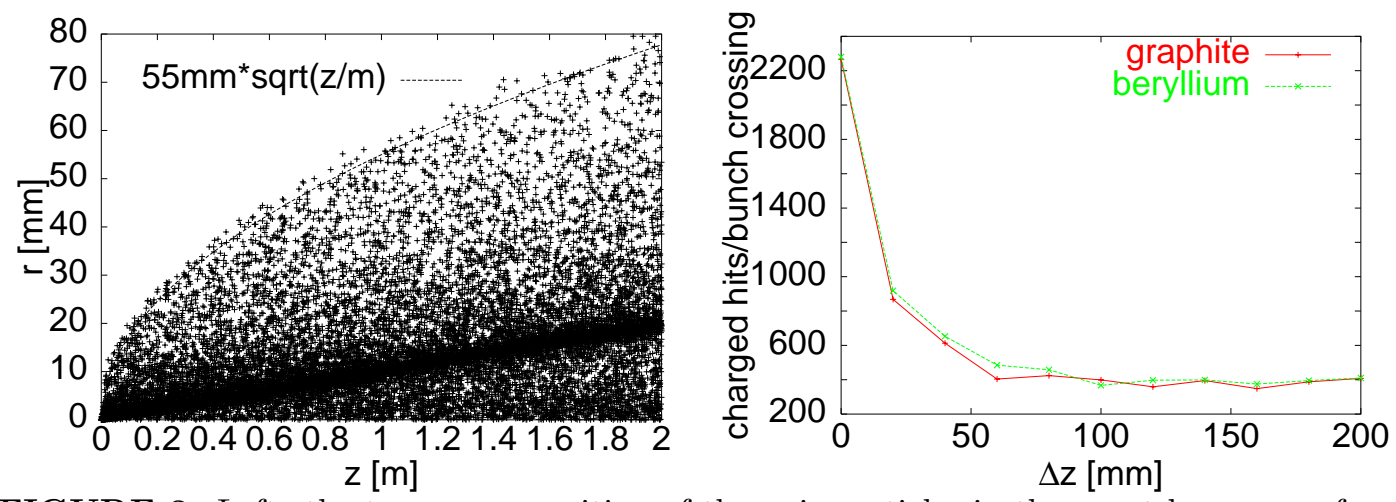

FIGURE 3. Left: the transverse position of the pair particles in the spent beam as a function of distance to the IP. Right: the number of hits in the innermost layer of the vertex detector for one bunch crossing as a function of the thickness of the inner mask layer.

created by the spent beam and from synchrotron radiation emitted by the incoming beams. It consists of a layer of instrumented tungsten covered by a layer of graphite (or any other low-Z material) towards the interaction point [3], see Fig. 2. While the tungsten shields the vertex detector from background coming into the detector, the graphite prevents the backscattering of low energy particles. In the instrumented mask, one can tag high energy particles down to $\theta_{0} \approx 40 \mathrm{mrad} \approx 55 \mathrm{~mm} \sqrt{1.9} / 1.9 \mathrm{~m}$, before being disturbed by the bulk of the particles.

To evaluate the mask, a simulation with all incoherent pairs of one bunch crossing was performed. The thickness $\Delta z$ of the layer of low-Z material was varied. The number of hits from backscattered particles is reduced to a negligible level at about $\Delta z=6 \mathrm{~cm}$, see right-hand side of Fig. 3. The spent beam line will have to be carefully designed to avoid that particles are scattered back through the spent beam hole in the inner mask. The incoherent pairs deposit about $50 \mathrm{TeV}$ of energy in each of the inner masks per bunch crossing, the coherent pairs about $200 \mathrm{TeV}$. Currently, the statistical uncertainty of the latter value is quite large, 
since only a very small fraction of the simulated coherent pairs hit the mask.

The background in the central tracker due to incoherent pairs has been simulated using simple scoring planes at different radii $r$. The number of photons per train that cross these planes are $6 \times 10^{5}$ at $r=0.3 \mathrm{~m}$ and $3 \times 10^{5}$ at $r=1.8 \mathrm{~m}$.

Also the hadronic background has been simulated using HADES. The average visible energy per bunch crossing is about $90 \mathrm{GeV}$. The highest density of charged hits in the vertex detector caused by the hadronic background is about $0.25 \mathrm{~mm}^{-2}$ per bunch train. This is smaller than the hit density due to incoherent pairs but not completely negligible. Secondary neutrons have been simulated neglecting the backscattering from the calorimetry. The maximum flux at $r=0.3 \mathrm{~m}$ is found to be $3 \times 10^{9}$ per year of operation. It is highest around the masks and significantly smaller around the IP.

\section{CONCLUSION}

A preliminary mask design for the CLIC detector exists. The background level in the central tracker and in the vertex detector due to incoherent pair creation and hadron production has been calculated and seems acceptable. With CALYPSO the luminosity spectrum and with HADES the hadronic background can be included in event generators. This allows to investigate their impact on physics analyses.

\section{REFERENCES}

1. The CLIC Study Team, G. Guignard (ed). "A $3 \mathrm{TeV} \mathrm{e}^{+} \mathrm{e}^{-}$Linear Collider Based on CLIC Technology". CERN 2000-008 (2000).

2. P. Chen and K. Yokoya. "Beam-Beam Phenomena in Linear Colliders". KEKPreprint-91-002 (1991)

3. D. Schulte. "Study of Electromagnetic and Hadronic Background in the Interaction Region of the TESLA Collider". Phd-thesis, TESLA 97-08 (1997).

4. D. Schulte. "Background at Future Linear Colliders". in Workshop on the Development of Future Linear Electron-Positron Colliders edited by G. Jarlskog, U. Mjörnmark and T. Sjöstrand, Lund, 1999, pp. 59-73 and CERN/PS 1999-066.

5. R. Assmann, G. Guignard, O. Napoly, D. Schulte, F. Zimmermann. "Final Focus System for CLIC". CERN-SL-2000-057 (2000).

6. D. Schulte. "Simulation of an Intra-Pulse Interaction-Point Feedback for Linear Colliders". CERN/PS 2000-051 (AE).

7. D. Schulte. "High-Energy Beam-Beam Effects in CLIC". CERN/PS 99-017 (LP) (1999).

8. A copy can be obtained from the author (Daniel.Schulte@cern.ch).

9. D. Schulte and I. Syratchev. "Beam Loading Compensation in the Main Linac of CLIC". CERN/PS- 2000-050 (AE) (2000).

10. See $[8]$

11. C. Damerell. Private communication. 1994-2000. 